\title{
MULTIVARIABLE LQG OPTIMAL CONTROL - RESTRICTED STRUCTURE CONTROL FOR BENCHMARKING AND TUNING
}

\author{
David Greenwood, Michael A. Johnson, Michael J. Grimble \\ Industrial Control Centre, University of Strathclyde, Glasgow, Scotland, U. K. \\ Email: m.johnson@eee.strath.ac.uk Fax Number: 01415484203
}

Keywords Process control, controller assessment, optimal LQG control, restricted structure

\begin{abstract}
The paper introduces the benchmarking of multivariable systems using an offline optimal LQG approach. Polynomial system optimal LQG control theory is used to derive a solution to the multivariable restricted structure controller computation. This solution theory is then developed into an computational algorithm. The control problems of the hotstrip finishing mill interstand looper as found in the steel industry are used to demonstrate the multivariable controller benchmarking and restricted structure procedure developed.
\end{abstract}

\section{INTRODUCTION}

Single loop controller assessment techniques have developed to the point where online procedures have been used in industrial tests (Desborough and Harris, 1992; Thornhill et al, 1999). For multivariable controller assessment, the situation is not so forward. At the close of the monograph due to Huang and Shah (1999) there is strong indication that a successful multivariable procedure would probably be based on optimal control theory. Ideally, such a procedure should have an unconstrained optimal benchmark value, a benchmark value for the actual industrial control structure but with optimised parameters and a benchmark value for the industrial controller that has been implemented. Furthermore, the benchmarks should be calculated directly from process data.

However, the multivariable controller assessment problem also has other features not present in the single loop controller problem. Industrial multivariable controllers have an internal structure with two components; first, an input-output (matrix) structure which determines controller elements are zero and which will have a controller element and, a second structure where the actual form of the controller element itself is determined(for example, a P, PI or a PID controller element). Thus there is a need to be able to assess the existing industrial controller against potential options available in the multivariable controller structure. This paper uses an optimal control restricted structure formulation to address this extra structural feature of the multivariable controller assessment problem. For simplicity, denote benchmark cost function as, $J$ and the internal structure of restricted controller $K_{R S}$ by the symbol, $S_{l}$ so that the corresponding controller is, $K_{R S}\left(S_{l}\right)$, then the restricted structure optimisation problem can be given as, $\min _{\text {w.r.t. } K_{R S}\left(S_{l}\right)} J\left(S_{l}\right)$ and with $K_{R S}\left(S_{l}\right)$ closed loop stabilising.

If the optimal value of the restricted structure optimisation problem for structure $S_{l}$ is denoted $J^{o p t}\left(S_{l}\right)$, then the optimal cost function ordering for (say) a set of controller structures $S_{l 1} \subset S_{l 2} \subset S_{l 3}$ will be,

$$
J^{o p t} \leq J^{o p t}\left(S_{l 3}\right) \leq J^{o p t}\left(S_{l 2}\right) \leq J^{o p t}\left(S_{l 1}\right)
$$

This can be used in a benchmark inequality as,

$$
1 \leq \frac{J^{o p t}\left(S_{l 3}\right)}{J^{o p t}} \leq \frac{J^{o p t}\left(S_{l 2}\right)}{J^{o p t}} \leq \frac{J^{o p t}\left(S_{l 1}\right)}{J^{o p t}}
$$

As can be seen the multivariable controller assessment problem comprises several different requirements. A key one is to establish an optimal control framework for the comparison of different restricted structure multivariable controllers. A companion paper by Greenwood et al (2003) further presents a philosophy for different types of benchmarking problems in process control and also contributes to this work on the controller assessment problem.

\subsection{Layout and contributions of the paper}

In section 2 of the paper, the LQG based restricted structure controller computation principles are given. This is the first time the polynomial system solution of the socalled multivariable restricted structure problem has been presented. In section 3 , the new multivariable restricted structure controller procedure is used on the example of tension and angle control in a hotstrip rolling mill interstand looper unit. Typically the industrial solution is decentralised PID feedback control tuned manually. The paper reports some results from using the polynomial 
numerical algorithms to compute benchmark values. Conclusions and references close the paper.

\subsection{MULTIVARIABLE RESTRICTED STRUCTURE CONTROLLER THEORY AND ALGORITHMS}

The need for a multivariable benchmarking procedure motivates the theoretical development of this paper. A major assumption in this work is that the desired control design for an industrial control system can be captured adequately by LQG optimal control theory. A simple procedure to setup the LQG controller design parameters is presented by Greenwood (2003). The algorithm to compute the benchmark or optimal cost value for different industrial control structures but with optimised parameters is one which computes optimal restricted structure multivariable controllers. For this, new results are needed and presented here. Finally, the adoption of the LQG cost function as a benchmark ensures that the computation of the benchmark for the implemented industrial controller is a simple operation.

\subsection{The LQG optimal control method}

The theory is based on the continuous time, polynomial one degree of freedom multivariable system.

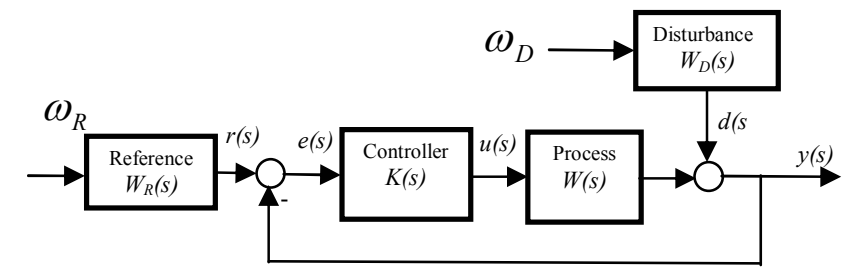

Figure 1 System configuration

Using Figure 1,

$\begin{array}{ll}\text { Plant } & y(s)=W(s) u(s)+d(s) \\ \text { Disturbance } & d(s)=W_{D}(s) \omega_{d}(s) \\ \text { Reference } & r(s)=W_{R}(s) \omega_{r}(s) \\ \text { Controller } & u(s)=K(s) e(s) \\ \text { Error } & e(s)=r(s)-y(s)\end{array}$

White noise signals, $\omega_{R}$ and $\omega_{D}$, are zero-mean with unit covariance matrix, and are mutually statistically independent. The system transfer functions are given a common denominator form, are coprime and may be written as,

$$
\left[\begin{array}{lll}
W & W_{D} & W_{R}
\end{array}\right]=A^{-1}\left[\begin{array}{lll}
B & D & E
\end{array}\right]
$$

The benchmark is taken to be the steady state stochastic LQG quadratic cost function given by,
$J_{L Q G}=\frac{1}{2 \pi j} \int_{D}\left\{\operatorname{trace}\left\{Q_{c}(s) \Phi_{e e}(s)\right\}+\operatorname{trace}\left\{R_{c}(s) \Phi_{u u}(s)\right\}\right\} d s$

Where $\Phi_{e e}(s)$ and $\Phi_{u u}(s)$ are rational matrix spectral density transfer functions for the error $e(s)$ and the control $u(s)$. The integration is around the usual $\mathrm{D}$ contour. The multivariable dynamic weights are denoted $Q_{c}(s)$ and $R_{c}(s)$; these are used to shape the response produced by the controller. The fundamental framework for an LQG benchmark procedure requires a decomposition of the LQG cost function. Various polynomial system definitions are given first. The polynomial matrix representations of the error and control weights are, respectively,

$$
\begin{aligned}
& Q_{c}(s)=A_{q}^{-*} B_{q}^{*} B_{q} A_{q}^{-1}=Q_{c}^{*}(s) \\
& R_{c}(s)=A_{r}^{-*} B_{r}^{*} B_{r} A_{r}^{-1}=R_{c}^{*}(s)
\end{aligned}
$$

where, the polynomial matrices $A_{q}, B_{q}, A_{r}, B_{r}$ make $Q_{c} \geq 0$ and $R_{c}>0$ on the D-contour. The transfer function for the weighted system is,

$W_{W T}=A_{q}^{-1} A^{-1} B A_{r}=B_{1} A_{1}^{-1}$

The control polynomial spectral factor $D_{c}$ is from,

$Y_{c}^{*} Y_{c}=W^{*} Q_{c} W+R_{c}=\left[D_{c}\left(A_{r} A_{1}\right)^{-1}\right]^{*}\left[D_{c}\left(A_{r} A_{1}\right)^{-1}\right]$

with $D_{c}^{*} D_{c}=B_{1}^{*} B_{q}^{*} B_{q} B_{1}+A_{1}^{*} B_{r}^{*} B_{r} A_{1}$

Generalised spectral factor $Y_{f}$ is defined as,

$$
\begin{aligned}
\Phi_{T T} & =\Phi_{r r}+\Phi_{d d} \\
& =Y_{f} Y_{f}^{*}=\left[A^{-1} D_{f}\right]\left[A^{-1} D_{f}\right]^{*}
\end{aligned}
$$

and polynomial filter spectral factor $D_{f}$ obtains as,

$$
D_{f} D_{f}^{*}=E E^{*}+D D^{*}
$$

The following Diophantine equation solutions are needed in the optimal solution. Solve for triple $\left(H_{0}, G_{0}, F_{0}\right)$ with $F_{0}$ of smallest row degree,

$$
\begin{aligned}
& D_{c}^{*} G_{0}+F_{0} A_{2}=B_{1}^{*} B_{q}^{*} B_{q} D_{2} \\
& D_{c}^{*} H_{0}-F_{0} B_{2}=A_{1}^{*} B_{r}^{*} B_{r} D_{3}
\end{aligned}
$$

With right co-prime decompositions,

$$
A_{2} D_{2}^{-1}=D_{f}^{-1} A A_{q} \text { and } B_{2} D_{3}^{-1}=D_{f}^{-1} B A_{r}
$$

\section{Theorem 1 Multivariable LQG benchmark cost values} The LQG benchmark cost function $J_{L Q G}$ may be decomposed as follows,

$$
J_{L Q G}=J_{A}+J_{B}+J_{C}
$$


$J_{A}=\frac{1}{2 \pi j} \int_{D} \operatorname{trace}\left\{X_{A}^{*} X_{A}\right\} d s$

$J_{B}=\frac{1}{2 \pi j} \int_{D} \operatorname{trace}\left\{X_{B}^{*} X_{B}\right\} d s$

$J_{C}=\frac{1}{2 \pi j} \int_{D} \operatorname{trace}\left\{X_{C}\right\} d s$

and,

$$
\begin{aligned}
& X_{A}(s)=\left[H_{0} D_{3}^{-1} A_{r}^{-1} K_{n}-G_{0} D_{2}^{-1} A_{q}^{-1} K_{d}\right]\left(A K_{d}+B K_{n}\right)^{-1} D_{f} \\
& X_{B}(s)=-D_{C}^{-*}(s) F_{0}(s) \\
& X_{C}(s)=\left[Q_{C}-Q_{C} W^{*}\left(Y_{C}^{*} Y_{C}\right)^{-1} W Q_{C}\right] \Phi_{T T}
\end{aligned}
$$

Greenwood (2003) gives full details of the proof.

\section{Corollary 1 Optimal LQG benchmark cost values}

The optimal value of the multivariable LQG benchmark cost is given by,

$$
J_{L Q G}^{o p t}=J_{B}+J_{C}
$$

and the related optimal controller is derived as

$$
K_{\text {opt }}(s)=A_{r}(s) D_{3}(s) H_{0}^{-1}(s) G_{0}(s) D_{2}^{-1}(s) A_{q}^{-1}(s)
$$

Greenwood (2003) gives full details of the proof.

\section{Design procedure for optimal LQG controller}

After the main equations have been established, the second component of the multivariable LQG controller benchmark philosophy is a straightforward controller design problem setup. This means that simple multivariable controller tuning rules must be available in order to set up the benchmark cost problem. It is the structure of the optimal controller expression (18b) that indicates how these rules should be established and full details have been given by Greenwood (2003).

\subsection{Restricted structure benchmark cost theory}

It is now necessary to establish the benchmark cost for optimal values of the control structure actually used by the implemented industrial control system. In solving this problem the theory can also be used to generate the optimal fixed structure controller parameters and also investigate the effectiveness of different configurations for the restricted structure controller per se.

The development of the restricted structure cost function benchmark begins from the results in Theorem 1 and Corollary 1 from which it is easily shown that,

$$
J_{L Q G}=J_{A}+J_{B}+J_{C}=J_{A}+J_{L Q G}^{o p t}
$$

with

$$
J_{A}=\frac{1}{2 \pi j} \int_{D} \operatorname{trace}\left\{X_{A}^{*} X_{A}\right\} d s
$$

and where equation (17e) is re-written as,

$$
X_{A}(s)=\left[H_{0} D_{3}^{-1} A_{r}^{-1} K_{n} K_{d}^{-1}-G_{0} D_{2}^{-1} A_{q}^{-1}\right]\left(A+B K_{n} K_{d}^{-1}\right)^{-1} D_{f}
$$

The internal structure of a restricted structure controller has two components; a matrix structure which determines which controller elements are zero and which will have a controller element and a second structure where the actual form of the controller element itself will be determined(for example, a P, PI or a PID controller element). For simplicity, denote the internal restricted structure of $K_{R S}$ by the symbol, $S_{l}$ and denote the corresponding controller, $K_{R S}\left(S_{l}\right)$, then the LQG restricted structure optimisation problem can be given as,

$$
\begin{aligned}
& \min _{w . r . t . K_{R S}\left(S_{i}\right)} J_{R S}\left(S_{l}\right)=\frac{1}{2 \pi j} \int_{D} \operatorname{trace}\left\{X_{A}^{*} X_{A}\right\} d s \\
& \text { with } X_{A}(s)=\left[H_{0} D_{3}^{-1} A_{r}^{-1} K_{R S}\left(S_{l}\right)-G_{0} D_{2}^{-1} A_{q}^{-1}\right]\left(A+B K_{R S}\left(S_{l}\right)\right)^{-1} D_{f} \\
& \text { and } K_{R S}\left(S_{l}\right) \text { closed loop stablising }
\end{aligned}
$$

The calculation for the optimal restricted structure cost function value begins by assuming that a suitable set of LQG cost function weighting matrices have been selected and that these capture the essence of an ideal desired design. Based on the given LQG cost function, it is then assumed that the matrices $D_{f}, H_{0}, G_{0}, D_{2}, D_{3}$ associated with the full optimal solution have been computed using the appropriate equations of Section 2.1.

Denote the selected restricted structure controller as $K_{R S}^{0}$ and its related vector of controller parameters as $\theta^{0} \in \mathfrak{R}^{n_{c}}$ where the number of parameter is given by $n_{c}$. The restricted structure optimisation problem in the equation of (20) is nonlinear in the restricted structure parameters and the optimal solution is found from an iterative scheme based on the scalar template algorithm due to Grimble (2000). The starting point for this algorithm is a known closed loop stable industrial controller specified through the vector of controller parameters, $\theta^{0} \in \mathfrak{R}^{n_{c}}$. The iterative step of the new algorithm is given by the following equations.

\section{Multivariable LQG restricted structure iteration}

Let the number of restricted controller parameters be $n_{c}$ and let $k$ be an iteration index. Denote the $k^{\text {th }}$ closed loop 
restricted structure controller as $K_{R S}^{k}$ and let its related vector of controller parameters be $\theta^{k} \in \mathfrak{R}^{n_{c}}$, so that:

$$
X_{A}(j \omega)=X_{R}\left(\omega \mid \theta^{k}\right)+j X_{I}\left(\omega \mid \theta^{k}\right)
$$

and hence,

$$
\begin{aligned}
& J_{R S}\left(S_{i}\right)=\frac{1}{2 \pi j} \int_{D} \operatorname{trace}\left\{X_{A}^{*} X_{A}\right\} d s \\
& =\frac{1}{\pi} \int_{0}^{\infty}\left\{\theta^{T} P\left(\omega \mid \theta^{k}\right) \theta+2 Q\left(\omega \mid \theta^{k}\right) \theta+C\left(\omega \mid \theta^{k}\right)\right\} d \omega \\
& =\theta^{T} \widetilde{P}\left(\theta^{k}\right) \theta+2 \widetilde{Q}\left(\theta^{k}\right) \theta+\widetilde{C}\left(\theta^{k}\right)
\end{aligned}
$$

Then a $(k+1)$ th update of restricted structure controller $K_{R S}$ is given by, $\theta^{k+1} \in \mathfrak{R}^{n_{c}}$,where,

$\theta^{k+1}=-\left[\widetilde{P}\left(\theta^{k}\right)\right]^{-1} \widetilde{Q}\left(\theta^{k}\right)$

and the updated optimal restricted cost function value is, $J_{R S}^{k+1}\left(S_{l}\right)=\widetilde{C}\left(\theta^{k}\right)-\widetilde{Q}\left(\theta^{k}\right)^{T}\left[\widetilde{P}\left(\theta^{k}\right)\right]^{-1} \widetilde{Q}\left(\theta^{k}\right)$

Greenwood (2003) gives full details of the nontrivial derivation for the equations (21a-21d).

\section{Algorithm 1 Optimal Multivariable LQG Restricted Structure Controller \\ Step 1 Initialisation}

Determine a set of LQG cost function weighting matrices for the desired design and solve the LQG equations to determine the matrices $D_{f}, H_{0}, G_{0}, D_{2}, D_{3}$

Define the controller, $K_{R S}$ and $\theta \in \mathfrak{R}^{n_{c}}$

Define $K_{R}(\omega)=\sum_{i=1}^{n_{c}} E_{R i} \theta_{i}$ and $K_{I}(\omega)=\sum_{i=1}^{n_{c}} E_{\mathrm{I} i} \theta_{i}$

Set $k=0$

Define a convergence tolerance, tol, for $\left\|\theta^{k+1}-\theta^{k}\right\|_{2}$

Define initial controller values, $K_{R S}^{0}$ and $\theta^{0} \in \mathfrak{R}^{n_{c}}$

Step 2 Loop Step

Determine $\widetilde{P}\left(\theta^{k}\right)=\frac{1}{\pi} \int_{0}^{\infty} P\left(\omega \mid \theta^{k}\right) d \omega$,

$\widetilde{Q}\left(\theta^{k}\right)=\frac{1}{\pi} \int_{0}^{\infty} Q\left(\omega \mid \theta^{k}\right) d \omega, \widetilde{C}\left(\theta^{k}\right)=\frac{1}{\pi} \int_{0}^{\infty} C\left(\omega \mid \theta^{k}\right) d \omega$

Solve $\theta^{k+1}=-\left[\widetilde{P}\left(\theta^{k}\right)\right]^{-1} \widetilde{Q}\left(\theta^{k}\right)$

Evaluate

$J_{R S}^{k+1}\left(S_{l}\right)=\widetilde{C}\left(\theta^{k}\right)-\widetilde{Q}\left(\theta^{k}\right)^{T}\left[\widetilde{P}\left(\theta^{k}\right)\right]^{-1} \widetilde{Q}\left(\theta^{k}\right)$

Step 3 Convergence step

If $\left\|\theta^{k+1}-\theta^{k}\right\|_{2}<$ tol then stop with $\theta^{o p t}=\theta^{k+1}$ and

$J_{R S}^{o p t}\left(S_{l}\right)=J_{R S}^{k+1}\left(S_{l}\right)$ else $k:=k+1$, goto Step 2
Comments on Algorithm 1: The numerical experience with the routine has been that it is very robust in terms of convergence. This behaviour of the multivariable controller routine reflects that of the scalar procedure reported by Grimble (2000).

\subsection{HOTSTRIP FINISHING MILL APPLICATION}

\subsection{The hotstrip finishing mill looper system}

The hotstrip finishing mill is a tandem mill of six or seven stands. Each rolling stand is usually a four-high rolling stand. The purpose of the roll stands is to effect a gauge (thickness) reduction in the strip as it travels through the mill. Between each pair of stands is a looper arm as shown in Figure 2. The looper arm can be raised or lowered to maintain the steel strip in tension as it is rolled. The looper arm angle is about $15^{\circ}$, but this angle varies in reaction to tension disturbances and weight changes. The control of looper angle and strip tension is a multivariable problem with considerable process interaction.

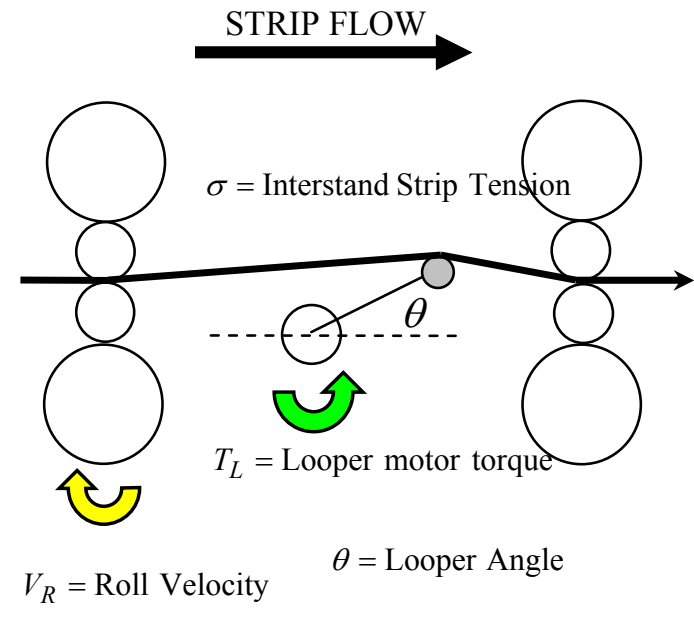

Figure 2 The hotstrip finishing mill looper system

\section{Looper Model}

A full description of the modelling of the finishing mill inter-stand looper system and a small signal linear looper system model was given by Johnson et al (1999). The model variables were interstand strip tension, $\sigma$, looper angle, $\theta$, roll velocity, $V_{R}$ and looper motor torque, $T_{L}$. The small signal model is,

$$
\left[\begin{array}{c}
\Delta \sigma \\
\Delta \theta
\end{array}\right]=\left[\begin{array}{ll}
G_{11} & G_{12} \\
G_{21} & G_{22}
\end{array}\right]\left[\begin{array}{l}
\Delta V_{R} \\
\Delta T_{L}
\end{array}\right]
$$

The physical variables under control in the looper system are strip tension and looper arm angle. The tension is measured in $\mathrm{N} / \mathrm{m}^{2}$ with numerical values of the order $10^{5}$, 
whereas angle is measured in degrees with a step change being typically, $\pm 2.5^{\circ}$. The zero frequency gains of the full order transfer function models reported by Johnson et al (1999) lie in a range from $3.1 \times 10^{-3}$ to $10^{8}$. This range of scaling was found to have serious detrimental effects in the polynomial routines of spectral factorisation and Diophantine equation solution. Spurious effects and incorrect results occurred so that it was necessary to construct a demonstration system based on the observed physical behaviour of the full system but without the extreme numerical range of the full transfer function model. The results presented in this section use this $(2 \times 2)$ demonstration looper system model,

$$
G_{L S}(s)=\left[\begin{array}{ll}
G_{11}(s) & G_{12}(s) \\
G_{21}(s) & G_{22}(s)
\end{array}\right]=\left[\begin{array}{cc}
\frac{-120}{s+1.2} & \frac{100 s+9}{\mathrm{~s}^{2}+9+9} \\
\frac{24}{s+1.2} & \frac{0.9 s+9}{\mathrm{~s}^{2}+9+9}
\end{array}\right]
$$

\section{Controller Assessment Design Setup}

The above basic system model, was augmented with additions models to complete the LQG system description.

Reference Model: $W_{R}(s)=\operatorname{diag}\left\{\frac{0.05}{s}, \frac{0.05}{s}\right\}$

Disturbance Models: $W_{D}(s)=\operatorname{diag}\left\{\frac{1}{10 s+1}, \frac{0.1}{s+1}\right\}$

Cost function weights: $B_{q}(s)=\operatorname{diag}\{s+9, s+11\}$

$$
\begin{aligned}
& A_{q}(s)=\operatorname{diag}\{s, s\}, B_{r}(s)=\operatorname{diag}\{s+40, s+10\} \\
& A_{r}(s)=\operatorname{diag}\{40,10\}
\end{aligned}
$$

\section{Performance assessment of industrial controllers}

The Table 1 shows optimal cost function values and the benchmark index for a few of the control structures. These are normalised with respect to the full optimal control cost value. The results of the table show that the off-diagonal controller structure is close to optimal and the Z-N tuning to be highly non-optimal. As a sample of the results possible, the response traces of Figures 2 and 3 show quite

\begin{tabular}{|c|c|c|}
\hline Case \& Control Structure & Cost Value & CPI \\
\hline \multicolumn{3}{|c|}{ Decentralised Structure ZN Tuning } \\
\hline $\mathrm{PI}(1,1) \mathrm{PI}(2,2)$ & 50018.35 & 53.36 \\
\hline \multicolumn{3}{|c|}{ Decentralised Restricted Structure } \\
\hline $\mathrm{PI}(1,1) \mathrm{PI}(2,2)$ & 1987.746 & 2.12 \\
\hline $\operatorname{PI}(1,2) \operatorname{PI}(2,1)$ & 1175.139 & 1.25 \\
\hline \multicolumn{3}{|c|}{ Limited Interactive Restricted Structure } \\
\hline PI(1,1) PI(2,1) PI(2,2) & 1927.816 & 2.06 \\
\hline \multicolumn{3}{|c|}{ Full Interactive Restricted Structure } \\
\hline $\mathrm{PI}(1,1) \mathrm{PI}(1,2) \mathrm{PI}(2,1) \mathrm{PI}(2,2)$ & 1110.798 & 1.18 \\
\hline \multicolumn{3}{|c|}{ Full Optimal LQG Control Structure } \\
\hline $\mathrm{K}(1,1) \mathrm{K}(1,2) \mathrm{K}(2,1) \mathrm{K}(2,2)$ & 937.346 & 1 \\
\hline
\end{tabular}
different mechanism at work in the two types of controller solutions.
The paper opened with a brief introduction to the generic features of benchmarking emphasising the desirability of an optimisation framework. This motivated the idea of benchmarking multivariable control systems using an offline optimal LQG approach. Polynomial system optimal LQG control theory was used to give an optimal cost calculation, and to provide a solution to the multivariable restricted structure control computation. The theory was then developed into an appropriate algorithm.

The industrial control problem of the hotstrip finishing mill interstand looper used in the steel industry was used to demonstrate the multivariable controller benchmarking and restricted structure procedure developed. Benchmark comparisons between a Ziegler-Nichols and restricted structure designs based on the optimal LQG index were shown. The quite different looper control mechanisms between a full optimal and a PI decentralised solution were shown. The results demonstrated the utility of quantifying the cost performance benefit obtained by introducing different levels of structural complexity into the multivariable controller.

\section{Acknowledgements}

The authors acknowledge the support of the United Kingdom's Engineering and Physical Science Research Council under grants GR/L98237(Steel project) and the Platform Grant (GR/R04683-01).

\section{REFERENCES}

Desborough, L. D. and T. J. Harris, 1992, Performance assessment measures for univariate feedback control, Canadian J. Chem. Engrg., Vol 70, 1186-1197

Greenwood D., 2003, PhD Thesis, University of Strathclyde, Glasgow, Scotland, UK. 
Grimble, M. J. 2000, Restricted Structure LQG Optimal Control for Continuous Systems, IEE Proc. Control Theory Applications, Vol 147, No2, (185-195), March

Grimble M. J., 2001, Restricted structure controller performance assessment and benchmarking, Proc. American Control Conference, 2718-2723.

Grimble M. J. and M. A. Johnson, 1988, Optimal Control and Stochastic Estimation, Volume $1 \& 2$, John Wiley and Sons, Chichester, UK.

Huang B and S. L. Shah, 1999, Performance Assessment of Control Loops, 255pp, ISBN 1-85233-639-0, 1999

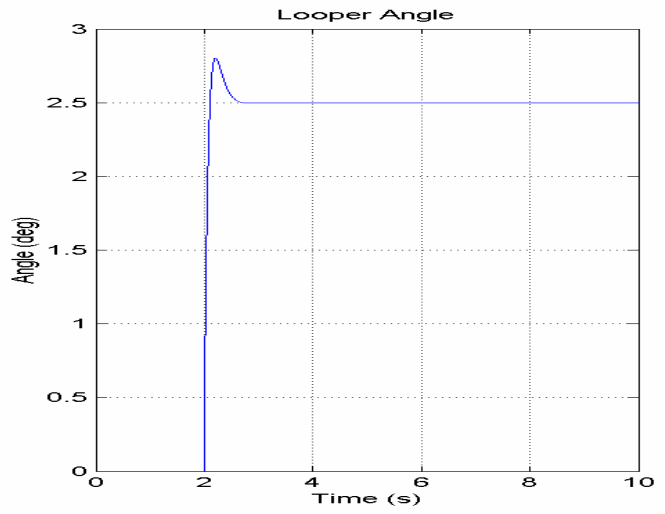

Johnson M. A., G. Hearns and T. Lee, 1999, The Hot Strip Rolling Mill Looper : A Control Case Study, Mechatronic Systems, Techniques And Applications(Editor: C. T. Leondes),

Gordon Breach Science Publishers, New Jersey, U. S. A., Volume 1, Industrial Manufacturing, Chapter 4, (169-253), October, ISBN 90-5699-670-3

Thornhill, N. F., M. Oettinger and P. Fedenczuk, 1999, Refinery Wide Control Loop Performance Assessment, Journal Process Control, Vol 9, 109-124.

Figure 2 Z-N Solution results for $2.5^{\circ}$ Looper angle reference change
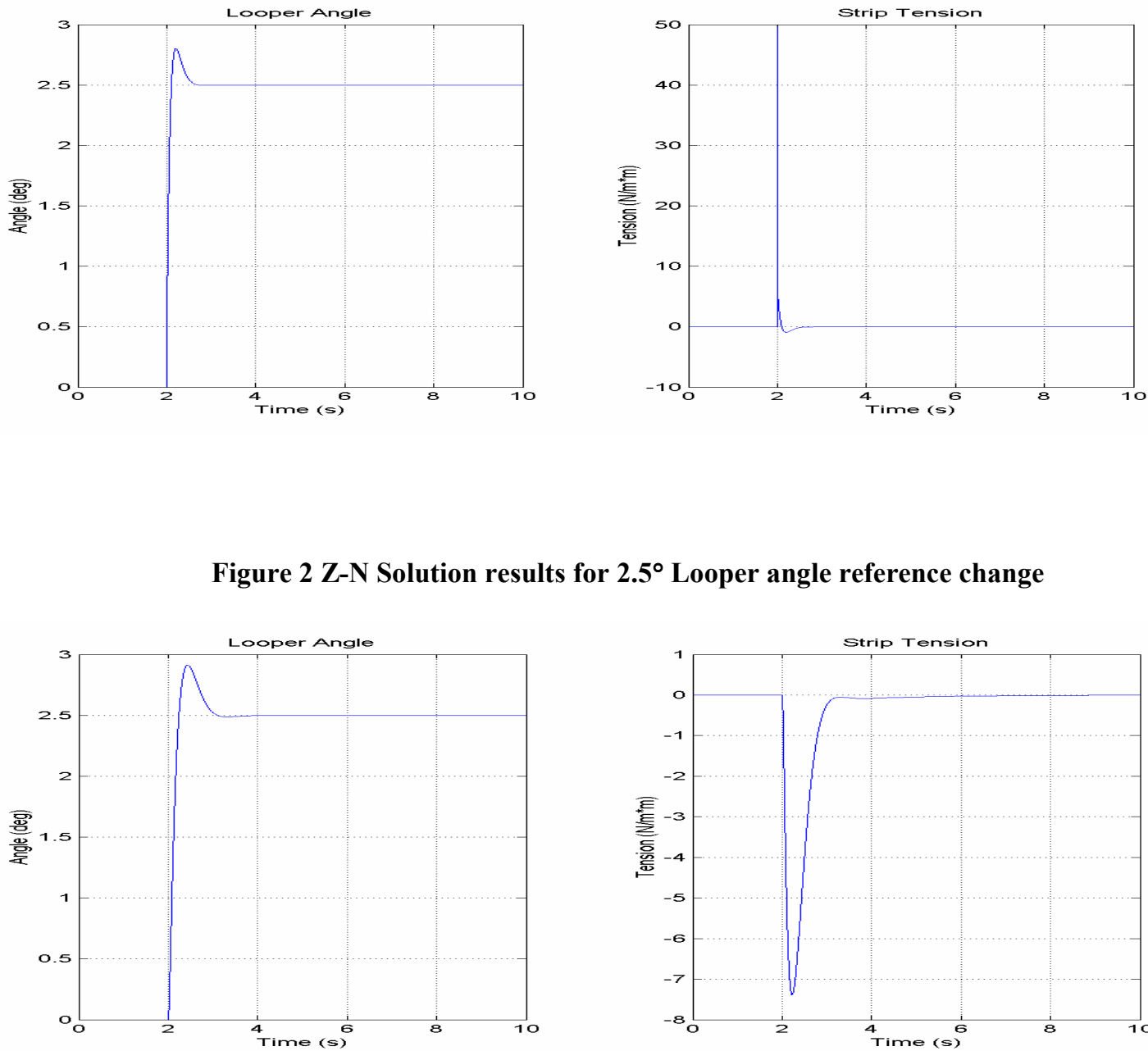

Figure 3 LQG Solution results for $2.5^{\circ}$ Looper angle reference change 